\title{
Caracterización de las unidades productoras de papa (Solanum tuberosum L.) en la provincia de Jauja, Junín, Perú
}

\author{
Characterization of potato producing units (Solanum tuberosum L.) \\ in the province of Jauja, Junín, Perú \\ Roberto Coaquira Incacari ${ }^{1}$, Alberto Julca Otiniano ${ }^{2}$ \\ Roberto Jhanfranco Coaquira Lastarria1, Juan Waldir Mendoza-Cortez ${ }^{2 *}$
}

\section{RESUMEN}

Es incuestionable la importancia que tiene el cultivo de papa en el Perú. Sin embargo, hay carencia de estudios que busquen entender la dinámica de los sistemas de producción de dicho cultivo, debido a la elevada diversidad social, cultural, económica y ambiental que caracteriza a cada región productora. En vista de ello, este trabajo tuvo como objetivo caracterizar las unidades productoras de papa (UPP) en los distritos de Acolla y San Lorenzo, pertenecientes a la provincia de Jauja, Junín, Perú. Los productores muestreados para este estudio fueron 97 (Acolla) y 93 (San Lorenzo), a quienes se les aplicaron encuestas estructuradas, considerando los factores sociales, económicos y ambientales. Inicialmente, se realizó el análisis estadístico descriptivo de los datos para eliminar variables con baja capacidad discriminatoria. Seguidamente, las variables seleccionadas fueron analizadas por métodos multivariados (análisis de componentes principales, análisis factorial y análisis de conglomerados) para la tipificación de las fincas. De acuerdo con los resultados obtenidos, se identificaron tres formas de gestión bien diferenciadas entre sí. Las nuevas variables o variables sintéticas estuvieron constituidas por el "recurso de capital social" con poder explicativo del 9,0\% sobre la varianza total (1 variable de 8\% de 16 UPP), el "recurso de capital financiero" con 8,3\% (2 variables de $48 \%$ de 92 UPP) y el "recurso de capital natural" con 7,2\%, (1 variable de 26\% de 49 UPP). Según las variables sintéticas, el "recurso de capital financiero" fue el conjunto mayoritario de pequeños y medianos productores, quienes transforman la papa con el fin de darle valor agregado y además realizan el almacenamiento de alimentos para su seguridad alimentaria, caracterizándose como agricultores que tienen una vasta experiencia rural-andina.

Palabras clave: análisis multivariado, sustentabilidad, tipificación de fincas.

\section{ABSTRACT}

The importance of potato farming in Peru is unquestionable. However, there is a lack of studies that endeavor to understand of the production systems of this crop due to the high social, cultural, economic, and environmental diversity that characterizes each producing region. Under this, the objective of this work was to characterize the potato producing units (UPP) in the districts of Acolla and San Lorenzo, belonging to the province of Jauja, Junin, Peru. The number of producers sampled for this study was 97 (Acolla) and 93 (San Lorenzo), to whom structured surveys were applied, considering the social, economic, and environmental factors. Initially, the descriptive statistical analysis of the data was done to eliminate variables having low discriminatory capacity; then, the selected variables were analyzed by multivariate methods (main components, factorial analysis, and cluster analysis) for the typification of the farms. According to the obtained results, three different management forms were identified. The new variables or synthetic variables were constituted by the "social capital resource" with explanatory power of $9.0 \%$ on the total variance (1 variable of $8 \%$ of 16 UPP), the "financial capital resource" with $8.3 \%$ (2 variables of $48 \%$ of 92 UPP) and the "natural capital resource" with $7.2 \%$, (1 variable of $26 \%$ of 49 UPP). According to the synthetic variables, the "financial capital resource" was the majority of small and medium producers, who transform the potato to provide added value and also store food store food to ensure a supply, characterizing themselves as farmers who have a vast rural-Andean experience.

Keywords: multivariate analysis, sustainability, farm typification.

1 Escuela de Posgrado, Universidad Nacional Agraria La Molina, Av. La Molina, s/n, CP 15024, La Molina, Lima, Perú.

2 Departamento de Fitotecnia, Facultad de Agronomía, Universidad Nacional Agraria La Molina, Av. La Molina, s/n, CP 15024, La Molina, Lima, Perú.

* Autor para correspondencia: jmendoza@lamolina.edu.pe

Fecha de Recepción: 17 de Marzo, 2019.

Fecha de Aceptación: 3 Mayo, 2019. 


\section{Introducción}

A nivel mundial, la papa tiene gran importancia económica y social por ser el cuarto cultivo más consumido en el mundo, luego del maíz, trigo y arroz (FAOSTAT, 2019). En el Perú, la papa es el tercer cultivo más sembrado (319.712 ha) y el segundo con mayor producción $(4.776,294$ t), destacando las regiones de Puno (742.924 t), Huánuco (668.370 t), La Libertad (466.632 t), Apurímac (411.958 t), Cuzco (388.467 t) y Junín (365.684 t). Adicionalmente, 710 mil familias dependen de este cultivo, el cual emplea 33,4 millones de jornales, lo que representa alrededor del 4,0\% del PBI agrícola (MINAGRI, 2017).

Actualmente, los diferentes sistemas de producción agropecuarios enfrentan dificultades debido al proceso de la globalización. Por consiguiente, es necesario realizar estudios que busquen entender y mejorar la gestión de dichos sistemas, para poder hacer frente a los diversos cambios que están ocurriendo, con el objetivo de mantener un nivel de competitividad sostenible (Bravo-Ureta, 2008).

Entre las diversas herramientas que sirven para estudiar los sistemas de producción agropecuarios destaca la caracterización. Esta es una actividad preliminar necesaria para diagnosticar y analizar dichos sistemas, tanto a nivel de finca como de región (Escobar y Berdegué, 1990). En ese sentido, son esenciales los trabajos que buscan caracterizar los sistemas de producción agropecuarios, ya que esta actividad es fundamental para recabar información técnica sobre las prácticas productivas, entender el proceso de toma de decisión de los productores, identificar los principales factores limitantes (físicos, biológicos, sociales y económicos) y diseñar estrategias para solucionar problemas que afectan la estructura de producción y el desempeño económico de las propiedades rurales (León-Velarde y Barrera, 2003).

Oliveira et al. (2007) enfatizaron la necesidad de realizar estudios regionalizados, principalmente al considerar el carácter dinámico inherente al ambiente de producción con elevada diversidad socioeconómica, cultural y ecológica que distingue a la actividad agropecuaria. Por esta razón, es importante caracterizar las fincas productoras de papa en su específica región de cultivo (Rodríguez et al., 1990; Contreras y García, 2016), para formar grupos, establecer las diferencias entre ellas y definir sus principales características.
Trabajos actuales sobre la caracterización de diferentes sistemas de producción en general o de cultivos específicos, en condiciones agroecológicas y socioeconómicas diversas, realizados por investigadores como Leite et al. (2014), BenítezGarcía et al. (2015), Criollo-Escobar et al. (2016), Santos (2017), Verdezoto-Vargas y Vieria-Pico (2018) y Aquino et al. (2018), muestran la importancia de continuar haciendo este tipo de estudios.

Por lo expuesto, esta investigación se realizó con el objetivo de caracterizar las diferentes unidades productoras del cultivo de papa en la provincia de Jauja, Junín, Perú.

\section{Materiales y métodos}

El estudio se hizo en los distritos de Acolla

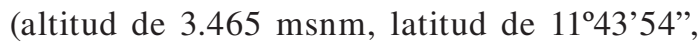
longitud de $75^{\circ} 32^{\prime} 56^{\prime}$ " y superficie de $122,4 \mathrm{~km}^{2}$ ) y San Lorenzo (altitud de $3.308 \mathrm{msnm}$, latitud de $11^{\circ} 50^{\prime} 50^{\prime}$, longitud de $75^{\circ} 23^{\prime} 03^{\prime \prime}$ y superficie de $22,15 \mathrm{~km}^{2}$ ), ubicados en la provincia de Jauja, región Junín.

A partir de la estructura que contenía la encuesta relacionada con los aspectos socioeconómicos del agricultor y la finca y factores ambientales del predio (dimensión ambiental, social y económico), fue que se hizo el cuestionario con indicadores sensibles a las condiciones del agroecosistema para que sean fácilmente comprendidos por los agricultores (Machado et al., 2015).

Del total de las Unidades Productoras de Papa (UPP) de los distritos de San Lorenzo (UPP = 1233) y Acolla (UPP $=3032$ ), fueron muestreados, conforme metodología de Scheaffer et al. (1986), 93 UPP y 97 UPP, respectivamente, a los cuales se les aplicó la encuesta estructurada.

Se definieron 42 variables originales, las cuales fueron codificadas para su procesamiento y análisis. La información recolectada fue sistematizada en hojas del Software Excel y analizada con el Software estadístico SPSS-V23.

Durante el procesamiento de los datos, primeramente, se calcularon los coeficientes de variación $(\mathrm{CV})$ para las 42 variables, de acuerdo con Escobar y Berdegué (1990). En este caso, las variables que tuvieron $\mathrm{CV}<30 \%$ fueron descartadas por carecer de poder discriminatorio. Seguidamente se realizó el análisis de las variables mediante métodos multivariados, los cuales buscan eliminar 
variables menos representativas, simplificando así modelos estadísticos en los que el número de variables es grande y para comprender la relación entre ellas (Hair et al., 2006).

Dentro de los métodos multivariados se utilizó el análisis factorial y el análisis discriminante, mediante el criterio de uniformidad o igualdad de medias, para la reducción dimensional de las variables. Antes de efectuar el análisis factorial, se realizaron las pruebas KMO (Káiser-MeyerOlkin) (Kaiser, 1970) y de esfericidad de Bartlett (Barlett, 1950), para evaluar si el modelo factorial en su conjunto es significativo.

A continuación, como parte del análisis factorial, se hizo el análisis de componentes principales, para identificar tanto variables que tienen influencia sobre la conformación de grupos formados, como variables altamente correlacionadas entre sí. Para facilitar la interpretación de las variables se realizó la rotación ortogonal de los componentes (rotación Varimax), propuesta por Kaiser (1958).

Una vez terminado ese procedimiento se aplicó el análisis de conglomerados o clúster, con el objetivo de agrupar las variables con características homogéneas al interior de cada grupo y a la vez lograr la mayor heterogeneidad entre grupos. Estas variables fueron graficadas en un dendrograma. Para alcanzar dicho objetivo fue utilizado el método de Ward (Ward, 1963), que incluyó como medida de proximidad la distancia euclidiana (Hair et al., 2006), y según el cual, cuando haya una menor distancia entre las variables, habrá una mayor similaridad entre ellas y, por lo tanto, pertenecerán a un mismo grupo.

\section{Resultados y discusión}

De las 42 variables originales analizadas, no todas contribuyeron a la caracterización de las UPP. En ese sentido, se seleccionaron 20 variables con mayor poder discriminante, de acuerdo al coeficiente de variación, como se observa en la Tabla 1. Los coeficientes de variación estuvieron en el rango de $30 \%$ a $78 \%$, que correspondieron a las variables "nivel de educación del agricultor" y "tipo de organización", respectivamente.

En el análisis estadístico multivariado realizado, el coeficiente de validez KMO de adecuación de muestreo fue $0,721(p>0,05)$, indicando que las 20 variables han sido elegidas apropiadamente y, según la prueba de esfericidad de Bartlett, que reporta

Tabla 1. Variables evaluadas y seleccionadas según el coeficiente de variación.

\begin{tabular}{|c|c|c|c|c|}
\hline Variables & Código & Media & DS\& & $\mathrm{CV}^{\$}(\geq 30 \%)$ \\
\hline Tipo de organización & Tdo $6-S^{\#}$ & 2,16 & 1,69 & $78 \%$ \\
\hline Capacitación que recibe & Cqr $10-\mathrm{S}$ & 1,99 & 1,53 & $77 \%$ \\
\hline Preparación del terreno & Pdt 14-A & 1,62 & 1,20 & $74 \%$ \\
\hline Orientación de los surcos & Ods 16-A & 2,85 & 1,92 & $67 \%$ \\
\hline Incidencia de enfermedades & Ide 25-A & 1,37 & 0,89 & $65 \%$ \\
\hline Incidencia de plagas & Idp 24-A & 1,36 & 0,88 & $64 \%$ \\
\hline Valor agregado de la papa & Vdp 39-E & 1,86 & 0,94 & $51 \%$ \\
\hline Métodos de control de plagas & Mcp 20-A & 1,09 & 0,53 & $48 \%$ \\
\hline Pendiente predominante & Ppd 15-A & 3,57 & 1,39 & $39 \%$ \\
\hline Uso de semilla certificada & Usc $22-\mathrm{A}$ & 2,98 & 1,11 & $37 \%$ \\
\hline Número de personas que viven en el hogar & $\mathrm{Nph} \quad 1-\mathrm{S}$ & 3,37 & 1,22 & $36 \%$ \\
\hline Conocimiento sobre contaminación ambiental & Cca $28-A$ & 2,78 & 0,99 & $35 \%$ \\
\hline Sistema de riego & Ssr $17-\mathrm{A}$ & 1,33 & 0,47 & $35 \%$ \\
\hline Ingreso por otros cultivos & Ioc $32-\mathrm{E}$ & 4,10 & 1,38 & $34 \%$ \\
\hline Nivel de satisfacción del agricultor & Nsa $8-\mathrm{S}$ & 2,60 & 0,85 & $33 \%$ \\
\hline Almacenamiento de alimentos & Ada $30-\mathrm{E}$ & 2,34 & 0,76 & $32 \%$ \\
\hline Precio de venta en chacra & Pvc 36-E & 1,16 & 0,37 & $32 \%$ \\
\hline Gasto en pesticidas & Gdp 34-E & 1,15 & 0,36 & $31 \%$ \\
\hline Factores climáticos que afectan & Fca $27-A$ & 1,14 & 0,34 & $30 \%$ \\
\hline Nivel de educación del agricultor & $\mathrm{Nda} \quad 4-\mathrm{S}$ & 2,93 & 0,88 & $30 \%$ \\
\hline
\end{tabular}

\&DS: desviación estándar; ${ }^{\$} \mathrm{CV}$ : coeficiente de variación; \#S: dimensión social, A: dimensión ambiental; E: dimensión económica. 
una significación de 0,000 , se acepta la hipótesis nula $(\mathrm{p}<0,05)$, que demuestra la conveniencia de trabajar con el método factorial.

Por otro lado, para reducir aún más las variables, se hizo el análisis discriminante, mediante la prueba de uniformidad de medias sobre los grupos de las 20 variables elegidas en el paso anterior. En este caso, fueron seleccionadas 13 variables que tienen poder discriminante $(\mathrm{p}<0,05)$ (Tabla 2$)$.

Al realizar el análisis de componentes principales, se puede verificar que solamente fueron seleccionados cinco Componentes Principales (CP), los cuales explican el 61,187\% de la varianza total acumulada rotada (Tabla 3). La selección de componentes principales fue hecha según el criterio de Kaiser (consiste en suprimir los componentes con varianzas explicadas inferiores a 1) y de acuerdo al porcentaje de varianza (consiste en mantener los componentes que acumulan una varianza de por lo menos 60\%) (Hair et al., 2006).

Cada CP generado tiene varianza máxima no correlacionada con los demás componentes. Además, el $\mathrm{CP}_{1}$ es la combinación de variables que expresan la mayor varianza $(1,992)$. En este trabajo, el $\mathrm{CP}_{1}$ y el $\mathrm{CP}_{5}$ explican el $15,322 \%$ y $10,338 \%$, de la inercia total de la nube de puntos de la matriz seleccionada (Tabla 3).
Tabla 2. Resumen del análisis discriminante (valores F, lambda de Wilks y significancias) mediante la prueba de igualdad sobre las medias de los grupos de las variables seleccionadas.

\begin{tabular}{lcrl}
\hline Variables & Lambda de Wilks & \multicolumn{1}{c}{$\mathrm{F}$} & Significancia \\
\hline Tdo 6-S\& & 0,733 & 68,487 & $0,000^{*}$ \\
Cqr 10-S & 0,991 & 1,648 & 0,201 \\
Pdt14-A & 0,987 & 2,381 & 0,124 \\
Ods 16-A & 0,916 & 17,150 & $0,000^{*}$ \\
Ide 25-A & 0,970 & 5,905 & $0,016^{*}$ \\
Idp 24-A & 0,957 & 8,411 & $0,004^{*}$ \\
Vdp39-E & 0,712 & 75,931 & $0,000^{*}$ \\
Mcp 20-A & 1,000 & 0,003 & 0,958 \\
Ppd 15-A & 0,187 & 820,019 & $0,000^{*}$ \\
Usc 22-A & 1,000 & 0,037 & 0,848 \\
Nph 1-S & 0,890 & 23,201 & $0,000^{*}$ \\
Cca 28-A & 1,000 & 0,000 & 0,992 \\
Ssr 17-A & 0,514 & 177,564 & $0,000^{*}$ \\
Ioc 32-E & 0,838 & 36,341 & $0,000^{*}$ \\
Nsa 8-S & 0,933 & 13,544 & $0,000^{*}$ \\
Ada 30-E & 0,890 & 23,197 & $0,000^{*}$ \\
Pvc 36-E & 1,000 & 0,073 & 0,787 \\
Gdp 34-E & 0,873 & 27,463 & $0,000^{*}$ \\
Fca 27-A & 0,944 & 11,158 & $0,001^{*}$ \\
Nda 4-S & 0,997 & 0,637 & 0,426 \\
\hline
\end{tabular}

* Significativo a 5\% de probabilidad; \& S: dimensión social, A: dimensión ambiental; E: dimensión económica.

Tabla 3. Varianza y cargas factoriales en la matriz de componentes rotados de las variables evaluadas.

\begin{tabular}{lccccc}
\hline \multirow{2}{*}{ Variables seleccionadas } & \multicolumn{5}{c}{ Cargas factoriales } \\
\cline { 2 - 6 } & $\mathrm{CP}_{1}$ & $\mathrm{CP}_{2}$ & $\mathrm{CP}_{3}$ & $\mathrm{CP}_{4}$ & $\mathrm{CP}_{5}$ \\
\hline Tdo6-S\& & $-0,749^{\#}$ & $-0,063$ & $-0,055$ & $-0,040$ & 0,227 \\
Ods16-A & $-0,038$ & $-0,146$ & 0,487 & $-0,116$ & $-0,608^{\#}$ \\
Idp24-A & $-0,010$ & 0,097 & $0,707^{\#}$ & 0,235 & 0,159 \\
Ide25-A & 0,124 & 0,004 & $0,667^{\#}$ & $-0,111$ & $-0,038$ \\
Vdp39-E & $-0,541^{\#}$ & $0,581^{\#}$ & $-0,018$ & 0,097 & 0,080 \\
Ppd15-A & $0,576^{\#}$ & $-0,400$ & 0,335 & 0,313 & $-0,177$ \\
Nph1-S & $0,708^{\#}$ & $-0,134$ & $-0,053$ & 0,086 & 0,418 \\
Ssr17-A & 0,478 & $-0,171$ & 0,325 & 0,470 & $-0,279$ \\
Acc33-E & 0,013 & $-0,528^{\#}$ & 0,181 & 0,476 & 0,039 \\
Nsa8-S & $-0,173$ & 0,038 & 0,231 & $-0,120$ & $0,753^{\#}$ \\
Ada30-E & $-0,127$ & $0,787^{\#}$ & 0,047 & 0,085 & $-0,047$ \\
Gdp34-E & $-0,096$ & 0,007 & 0,051 & $-0,825^{\#}$ & 0,003 \\
Fca27-A & 0,046 & 0,475 & 0,104 & $-0,237$ & 0,188 \\
Varianza total o explicada & 1,992 & 1,706 & 1,509 & 1,404 & 1,344 \\
\% de la varianza explicada & $15,322 \%$ & $13,121 \%$ & $11,609 \%$ & $10,796 \%$ & $10,338 \%$ \\
$\%$ de la varianza acumulada & $15,322 \%$ & $28,443 \%$ & $40,053 \%$ & $50,849 \%$ & $61,187 \%$ \\
\hline
\end{tabular}

\#Carga factorial>0,5 (Hair et al., 2006); \& S: Dimensión social, A: Dimensión ambiental; E: Dimensión económica. 
De acuerdo con la carga factorial (indica la correlación de cada variable con cada componente) calculada en la matriz de componentes, las variables con cargas mayores a 0,5 son consideradas estadísticamente significativas, según Hair et al. (2006). Por tanto, de acuerdo con la Tabla 3, hubo correlación positiva del $\mathrm{CP}_{1}$ con $\mathrm{Ppd} 15-\mathrm{A}$ y Nph1-S, del $\mathrm{CP}_{2}$ con Vdp39-E y Ada30-E, del $\mathrm{CP}_{3}$ con Idp24-A y Ide25-A y del $\mathrm{CP}_{5}$ con Nsa8-S; y correlación negativa del $\mathrm{CP}_{1}$ con Tdo6-S y Vdp39-E, del $\mathrm{CP}_{2}$ con Acc33-E, del $\mathrm{CP}_{4}$ con Gdp34-E y del $\mathrm{CP}_{5}$ con Ods16-A.

Ante la obtención de resultados poco satisfactorios para interpretar los componentes principales, se procedió al análisis factorial, mediante la técnica de rotación ortogonal, de tipo varimax (Coronel de Renolfi y Ortuño-Pérez, 2005; Candell-Soto, 2017; Aquino et al., 2018), para encontrar nuevos factores que permitan explicar de mejor manera las variables en los componentes principales.

En este trabajo, los resultados de la rotación varimax definieron cinco factores que explican el $36,980 \%$ de la varianza acumulada (Tabla 4). No obstante, la variancia acumulada hasta el nivel del cuarto eje $\left(\mathrm{F}_{4}\right)$, que fue de $31,182 \%$, resulta estadísticamente suficiente para considerar y seleccionar, solamente, los ejes $\mathrm{F}_{1}, \mathrm{~F}_{2}, \mathrm{~F}_{3}$ y $\mathrm{F}_{4}$, como factores que permiten analizar el fenómeno de la variabilidad funcional del conjunto de fincas o unidades productivas estudiadas.

Asimismo, a través del cálculo de la carga factorial en la matriz de factores (Tabla 4), fueron consideradas para el análisis las variables que tuvieron carga factorial mayor a 0,5 , por ser significativas (Hair et al., 2006). En ese sentido, las variables seleccionadas se agruparon, únicamente, en tres factores $\left(\mathrm{F}_{1}, \mathrm{~F}_{2}\right.$ y $\mathrm{F}_{3}$ ) (Tabla 4). El $\mathrm{F}_{1}$ tuvo alta correlación positiva, solamente, con la variable Nph1-S y el $F_{3}$ con la variable Ssr17-A. Por otro lado, el $\mathrm{F}_{2}$ tuvo alta correlación positiva con las variables Vdp39-E y Ada30-E, caracterizándose como el factor más influyente y que explica mejor el problema de tipificación en las unidades productoras del cultivo de papa (Tabla 4).

Cada factor principal, formado en el paso anterior, es una variable sintética que fue renombrada en función de las variables iniciales que incorporan. En ese sentido, el $\mathrm{F}_{1}$ fue representado como una medida de "recurso de capital social" (dimensión social), el cual expresa el $9,014 \%$ de la varianza explicada; $\mathrm{el} \mathrm{F}_{2}$ como "recurso de capital financiero" (dimensión económica) que expresa el 8,304\% de la varianza explicada; y el $\mathrm{F}_{3}$ como "recurso de capital natural" (dimensión ambiental), que expresa el 7,163\% de la varianza explicada (Tabla 4).

Tabla 4. Varianza y cargas factoriales en la matriz de factores rotados de las variables evaluadas.

\begin{tabular}{lccccc}
\hline \multirow{2}{*}{ Variables seleccionadas } & \multicolumn{5}{c}{ Cargas factoriales } \\
\cline { 2 - 6 } & $\mathrm{F}_{1}$ & $\mathrm{~F}_{2}$ & $\mathrm{~F}_{3}$ & $\mathrm{~F}_{4}$ & $\mathrm{~F}_{5}$ \\
\hline Tdo6-S\& & $-0,468$ & 0,088 & $-0,101$ & $-0,123$ & 0,279 \\
Ods16-A & $-0,088$ & $-0,115$ & $-0,039$ & 0,399 & $-0,387$ \\
Idp24-A & 0,013 & 0,060 & 0,160 & 0,468 & 0,112 \\
Ide25-A & 0,080 & $-0,001$ & 0,003 & 0,437 & $-0,021$ \\
Vdp39-E & $-0,386$ & $0,545^{\#}$ & $-0,034$ & $-0,060$ & 0,186 \\
Ppd15-A & 0,442 & $-0,381$ & 0,391 & 0,386 & $-0,233$ \\
Nph1-S & $0,684^{\#}$ & $-0,173$ & 0,147 & $-0,028$ & 0,236 \\
Ssr17-A & 0,326 & $-0,135$ & $0,517 \#$ & 0,328 & $-0,324$ \\
Acc33-E & 0,033 & $-0,333$ & 0,424 & 0,130 & $-0,008$ \\
Nsa8-S & $-0,039$ & 0,085 & $-0,105$ & 0,075 & 0,491 \\
Ada30-E & $-0,086$ & $0,626^{\#}$ & $-0,088$ & 0,040 & 0,027 \\
Gdp34-E & $-0,086$ & 0,033 & $-0,469$ & $-0,021$ & 0,035 \\
Fca27-A & $-0,042$ & 0,232 & $-0,182$ & 0,038 & 0,140 \\
Varianza total o explicada & 1,172 & 1,080 & 0,931 & 0,871 & 0,754 \\
\% de la varianza explicada & $9,014 \%$ & $8,304 \%$ & $7,163 \%$ & $6,701 \%$ & $5,798 \%$ \\
\% de la varianza acumulada & $9,014 \%$ & $17,318 \%$ & $24,481 \%$ & $31,182 \%$ & $36,980 \%$ \\
\hline
\end{tabular}

\#Carga factorial > 0,5 (Hair et al., 2006); \&S: Dimensión social, A: Dimensión ambiental; E: Dimensión económica. 
La realización del análisis de conglomerados o clúster, que fue complementado gráficamente con el dendrograma, permitió identificar tres grupos o tipos similares de unidades productoras de papa, con respecto a las tres nuevas variables sintéticas (recurso de capital social, recurso de capital financiero y recurso de capital natural) que se obtuvieron con la rotación varimax, considerando la distancia euclidiana de 11,3 (Figura 1).

Como resultado del análisis clúster, se verifica que el factor $\mathrm{F}_{2}$ se consolida como el factor que tiene mayor cantidad de tipos, por ende, con mayor homogeneidad intratipos. Dicho factor, representado por la variable sintética "recurso de capital financiero", está constituido por un número importante de UPP agrupadas, que en este caso son 92 UPP (48\% del total), de las cuales 76 UPP pertenecen al distrito de Acolla (83\%) y 16 UPP al distrito de San Lorenzo (17\%). Por otro lado, los factores $F_{1}$ y $F_{3}$, en conjunto, agruparon 98 UPP que representan el 52\% del total.

De acuerdo con los resultados, la tipificación de grupos permitió describir los tipos de sistemas de producción de papa presentes en los distritos de Acolla y San Lorenzo, que están representados por las tres variables sintéticas clasificadas anteriormente.

El primer grupo $\left(\mathrm{F}_{1}\right)$, representado por la variable sintética "recurso de capital social", con poder explicativo de $9,014 \%$ de la varianza total, está compuesto mayoritariamente por 16 UPP
(8\% del total), donde las personas que viven en el hogar son 3. Este dato está de acuerdo con el último censo nacional de 2017 (INEI, 2018), donde se verifica que en la región Junín, de forma general, predominan hogares donde el número de integrantes varía de 3 a 4 personas.

En el segundo grupo $\left(\mathrm{F}_{2}\right)$, representado por la variable sintética "recurso de capital financiero", que explica el 8,304\% de la varianza total, predominan 46 UPP (24\% del total) que le dan valor agregado, solamente, al $20 \%$ de la producción total de papa. Además, en 53 UPP ( $28 \%$ del total), del total de alimentos almacenados, un $25 \%$ no están relacionadas con el cultivo de papa. Como se puede verificar, el grupo 2 es el conjunto mayoritario de pequeñas y medianas unidades productoras de papa con gran uniformidad dentro de su tipo, que se caracterizan por tener una vasta experiencia rural andina, utilizando tecnología tradicional y recursos disponibles de su entorno para la transformación de la papa (papa seca, tocosh y principalmente chuño), y a la vez almacenan otros productos (oca y mashua) diferentes a dicho cultivo para su seguridad alimentaria.

El tercer grupo $\left(\mathrm{F}_{3}\right)$, que está representado por la variable sintética "recurso de capital natural" (explica el 7,163\% de la varianza total), se caracteriza porque en la mayoría de UPP (49) que representan el 26\% del total, predomina el sistema de riego por gravedad. Este resultado es interesante para la zona de estudio, en vista de que el cultivo de

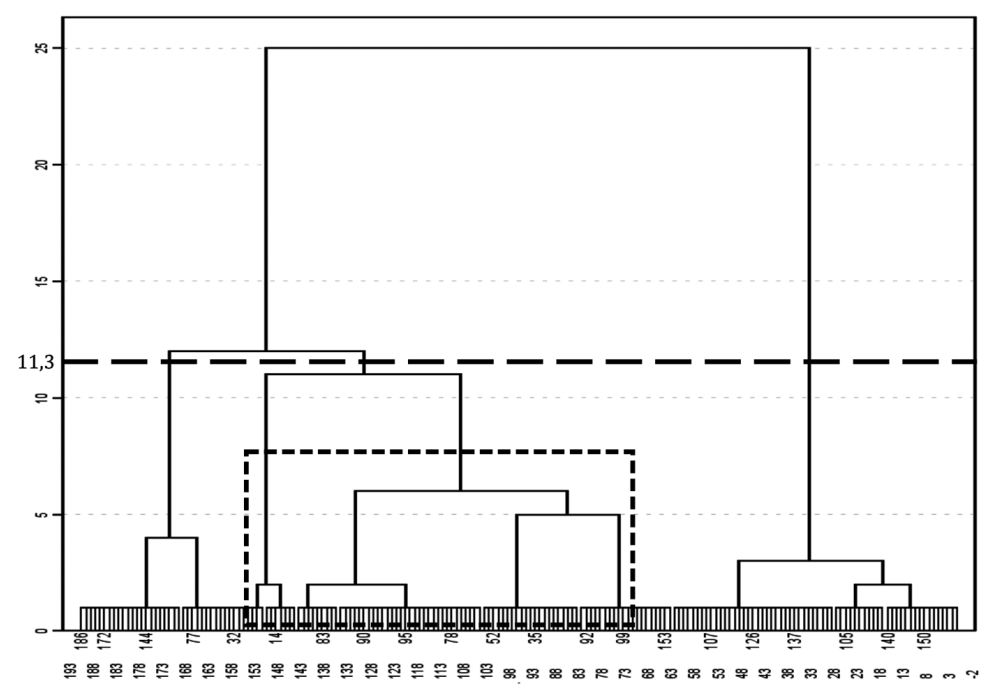

Figura 1. Dendrograma del análisis conglomerado o clúster, con el método de Ward (distancia de corte de 11,3 grupos o factores). 
papa en regiones localizadas a lo largo de la sierra (por encima de $2.000 \mathrm{msnm}$ ) se realiza en épocas de secano (MINAGRI, 2017).

Dentro de los pocos estudios existentes sobre la caracterización de los sistemas de producción de papa en el Perú, destaca el realizado por Contreras y García (2016) en tres distritos (Supe, Barranca y Pativilca) de la provincia de Barranca, Región Lima, en la costa peruana. Dichos autores, en contraste con lo observado en este trabajo que se hizo en regiones ubicadas por encima de los $3.000 \mathrm{msnm}$, constataron que la producción de papa en las tres localidades mostraron diferencias que dependen de las condiciones de producción imperantes en la Provincia. En ese sentido, las fincas ubicadas en las tres localidades son conducidas por productores que poseen servicios básicos pero no forman parte de organizaciones sociales y, además, predomina el monocultivo, la baja diversidad de especies y de cultivares de papa.

\section{Conclusiones}

La caracterización y tipificación de las unidades productoras de papa determinaron la existencia de tres grupos bien diferenciados entre sí. En el primer grupo, representado por la variable sintética "recurso de capital social", las personas que integran el hogar son 3 y constituyen el 8\% del total de unidades productoras de papa; el segundo grupo, representado por la variable sintética "recurso de capital financiero", está integrado por agricultores que le dan valor agregado, solamente, al $20 \%$ de la producción de papa y, además, almacenan un $25 \%$ de productos que son diferentes a la papa y constituyen el $48 \%$ del total de unidades productoras de papa; y el tercer grupo, representado por la variable sintética "recurso de capital natural", posee fincas con el sistema de riego por gravedad y constituyen el $26 \%$ del total de unidades productoras de papa.

\section{Literatura Citada}

Aquino, V.C.; Camarena, F.; Julca, A.; Jiménez, J.E.

2018. Caracterización multivariada de fincas productoras de tarwi (Lupinus mutabilis Sweet) del valle del Mantaro, Perú. Scientia Agropecuaria, 9 (2): 269-279.

Bartlett, M.S.

1950. Tests of significance in factor analysis. British Journal of Mathematical and Statistical Psychology, 3 (2): 77-85.

Benítez-García, E.; Jaramillo-Villanueva, J.L.; Escobedo-

Garrido, S.; Mora-Flores, S.

2015. Caracterización de la producción y del comercio de café en el Municipio de Cuetzalan, Puebla. Agricultura, Sociedad y Desarrollo, 12 (2): 181-198.

Contreras-Liza, S.; García-Bendezú, S.

2016. Caracterización socioeconómica del sistema de producción de papa en la provincia de Barranca. Big Bang Faustiniano, 5 (2): 37-41.

Coronel de Renolfi, M.; Ortuño-Pérez, S.F.

2005. Tipificación de los sistemas productivos agropecuarios en el área de riego de Santiago del Estero, Argentina. Problemas del Desarrollo. Revista Latinoamericana de Economía, 36 (140): 63-88.

Criollo-Escobar, H.; Lagos-Burbano, T.C.; Bacca-Ibarra, T.;

Muñoz-Belalcazar, J.A.

2016. Caracterización de los sistemas productivos de café en Nariño, Colombia. Revista U.D.C.A. Actualidad \& Divulgación Científica, 19 (1): 105-113.

Dixon, J.; Gulliver, A.; Gibbon, D.

2001. Sistemas de producción agropecuaria y pobreza: cómo mejorar los medios de subsistencia de los pequeños agricultores en un mundo cambiante. FAO, Banco Mundial. Roma, Italia. $50 \mathrm{p}$.

Escobar, E.; Berdegué, J.A.

1990. Conceptos y metodologías para tipificación de sistemas de finca: la experiencia de RIMISP (Red Internacional de
Metodologías de Investigación de Sistemas de Producción). En: Escobar, E.; Berdegué, J.A. (Eds.). Tipificación de sistemas de producción agrícola. Rimisp-Centro Latinoamericano para el Desarrollo Rural. Santiago, Chile. pp. 13-43.

FAO - Food and Agriculture Organization of the United Nations. 2019. Disponible: Faoestat. http://www.fao.org/faostat/ en/\#data. Consultado: 1/feb/ 2019.

Francisco, V.L.F. dos S.; Silva, P.R.; Ojima, A.L.R.; Baptistella, C. da S.L.; Verdi, A.R.

2008. Produção agrícola de uso intensivo da terra nas propriedades rurais do pólo frutícola bandeirante, estado do São Paulo: estudo de tipología. Revista de Economía Agrícola, 55 (2): 25-40.

Hair, J.F.; Black, W.C.; Babin, B.; Anderson, R. E.; Tatham, R.L. 2006. Multivariate data analysis. 6th ed. Pearson Education, London, UK. 688 p.

INEI - Instituto Nacional de Estadística e Informática.

2018. Perú: perfil sociodemográfico, informe nacional, censos nacionales: XII de población, VII de vivienda y III de comunidades indígenas. INEI. Lima, Perú. 641 p.

Leite, M.L. de M.V.; Silva, D.S. da; Andrade, A.P. de; Pereira, W.E.; Ramos, J.P. de F.

2014. Caracterização da produção de palma forrageira no Cariri Paraibano. Revista Caatinga, 27 (2): 192-200.

Kaiser, H.F.

1958. The varimax criterion for analytic rotation in factor analysis. Psychometrika, 23 (3): 187-200.

Kaiser, H.F.

1970. A second-generation little jiffy. Psychometrika, 35 (4): 401-15.

León-Velarde, C.U.; Barrera, V.H.

2003. Métodos bio-matemáticos para el análisis de sistemas agropecuarios en el Ecuador. INIAP, CIP. Quito, Ecuador. $187 \mathrm{p}$. 
Machado, V.M.; Nicholls, C.N.; Márquez, S.M.; Turbay, S. 2015. Caracterización de nueve agroecosistemas de café de la cuenca del río Porce, Colombia, con un enfoque agroecológico. IDESIA, 33 (1): 69-83.

MINAGRI - Ministerio de Agricultura y Riego.

2017. Papa: características de la producción nacional y de la comercialización en Lima metropolitana. Ministerio de Agricultura y Riego, Dirección General de Políticas Agrarias. Lima, Perú. 13 p.

Oliveira, A.S. de; Cunha, D. de N.F.V. da; Campos, J.M. de S.;

Vale, S.M.L.R. do; Assis, A.J. de.

2007. Identificação e quantificação de indicadores-referência de sistemas de produção de leite. Revista Brasileira de Zootecnia, 36 (2): 507-516.

Rodríguez, C.; Gallardo, J.; Fonseca, J.

1990. Tipificación de un grupo de fincas productoras de papa y cebolla en la zona norte de Cartago, mediante la técnica de análisis de factores. Agronomía Costarricense, 14 (2): 251-256.

Santos, M.J.P.L. dos.

2017. Principais tipologias de explorações agrícolas na União Europeia. Revista em Agronegócio e meio Ambiente, 10 (2): 273-290.

Scheaffer, R.L.; Mendenhall, W.; Lyman Ott, L.; Gerow, K.G. 1986. Elementary survey sampling. Duxbury Press. Boston, US. 324 p.

Verdezoto-Vargas, V.H.; Viera-Pico, J.E.

2018. Caracterización de sistemas de producción agropecuarios en el proyecto de riego Guarguallá-Licto, cantón Riobamba, provincia de Chimborazo. Ciencia y Tecnología, 11 (1): 45-53.

Ward, J.H., Jr.

1963. Hierarchical grouping to optimize an objective function. Journal of the American Statistical Association, 58 (301): 236-244. 